Gynäkologische Endokrinologie 2010 • 8:215-216 DOI 10.1007/s10304-010-0367-9

Online publiziert: 22. Juli 2010

๑) Springer-Verlag 2010

\section{Redaktion}

R. Felberbaum, Kempten

M. von Wolff, Bern

M. von Wolff · Z. Kollmann · A. Santi · E. Ocon · P. Stute Frauenklinik/Abteilung für Gynäkologische Endokrinologie und Reproduktionsmedizin, Universitätsfrauenklinik Bern, Schweiz

\title{
Ovartorsion nach In-vitro-Fertilisation
}

\section{Kasuistik}

Beschrieben wird eine 37-jährige Patientin mit primärer Sterilität. Im Rahmen einer IVF-Behandlung ovarielle Stimulation, Gewinnung von 14 Oozyten und Transfer von 2 Embryonen 2 Tage nach der Punktion.

Vier Tage nach der Punktion wacht die Patientin gegen 4 Uhr nachts mit starken Unterbauchschmerzen auf. Diese klingen zunächst wieder $a b$, werden dann aber zunehmend stärker und kolikartig. Um ca. 6 Uhr stellt sich die Patientin in der Notaufnahme eines Krankenhauses vor. Ultrasonographisch zeigt sich ein zystischer abdominaler Tumor, der über den Bauchnabel bis zum Leberrand reicht. Das linke Ovar ist leicht vergrößert, das rechte Ovar ist nicht sicher darstellbar.
Aufgrund des unklaren Befundes wird unverzüglich eine Längsschnittlaparotomie durchgeführt. Es zeigt sich ein massiv vergrößertes, torquiertes, eingeblutetes Ovar rechts. Um das Ovar vor die Bauchwand luxieren zu können (• Abb. 1), muss zuvor das intraovarielle Blut abgesaugt werden. Anschließend wird das Ovar problemlos abgesetzt. Das rechte Ovar ist leicht vergrößert, aber unauffällig.

\section{Diskussion}

Adnextorsionen treten mit einer Häufigkeit von $1 \mathrm{zu} 5000$ Schwangerschaften, überwiegend im 1. Trimenon auf. Das Risiko nach einer IVF-Behandlung wird auf ca. $0,1 \%$ geschätzt. Die rechte Seite ist häufiger als die linke Seite betroffen.
Abb. 1 - Zweifach torquiertes Ovar nach

Luxation vor der Bauchdecke

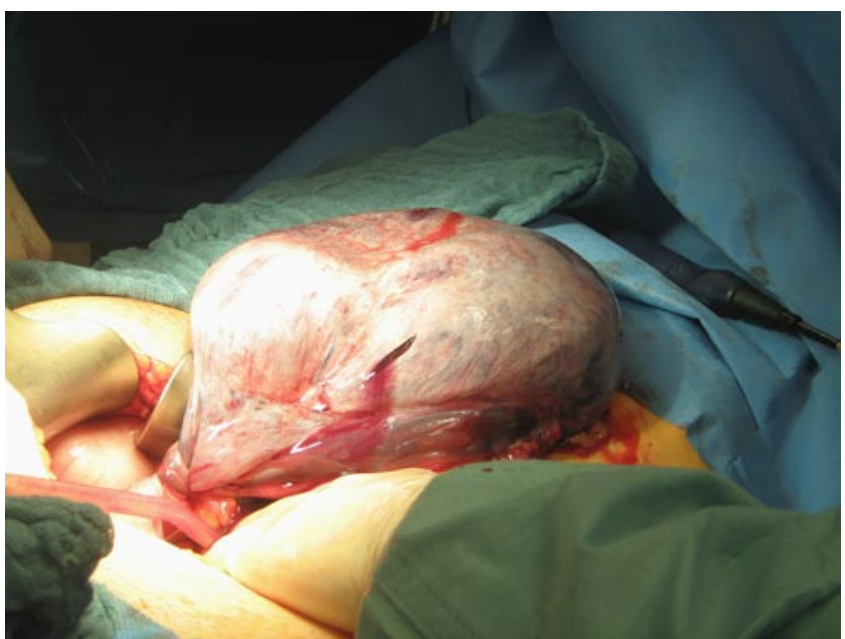


Gynäkologische Endokrinologie 2010 .

8:215-216

DOI 10.1007/s10304-010-0367-9

C) Springer-Verlag 2010

M. von Wolff · Z. Kollmann - A. Santi •

E. Ocon P. Stute

Ovartorsion nach

In-vitro-Fertilisation

\section{Zusammenfassung}

Das Risiko für das Auftreten einer Adnextorsion nach einer IVF-Behandlung wird auf ca. $0,1 \%$ geschätzt. Aufgrund der Seltenheit und des initial oft unauffälligen sonographischen Befundes wird eine Adnextorsion oft fehldiagnostiziert und die Behandlung verzögert. Die einzige effektive Therapie ist eine sofortige Laparoskopie, Retorsion und Verkleinerung des Ovars.

\section{Schlüsselwörter}

Ovartorsion · Adnextorsion · In-vitro-Fertilisation · IVF-Behandlung · Laparoskopie

\section{Ovarian torsion after in vitro fertilization}

\section{Abstract}

The risk for occurrence of adnexal torsion after IVF treatment is estimated to be about $0.1 \%$. Due to the rarity of the condition and often unremarkable initial ultrasound findings, an adnexal torsion is frequently misdiagnosed and treatment delayed. The only effective therapy is immediate laparoscopy, retorsion, and reducing the size of the ovary.

\section{Keywords}

Ovarian torsion · Adnexal torsion · In vitro fertilization · IVF treatment · Laparoscopy
Die Erfahrung zeigt, dass die Patientinnen häufig nachts im Schlaf symptomatisch werden. Möglicherweise liegt das Ovar nachts im Liegen nicht mehr fest im Becken und ist somit mobiler als in einer aufrechten Körperhaltung. Aufgrund der Seltenheit einer Adnextorsion und des initial oft unauffälligen sonographischen Befundes wird eine Adnextorsion oft fehldiagnostiziert und die Behandlung verzögert. Die einzige effektive Therapie ist eine sofortige Laparoskopie, Retorsion und Verkleinerung des Ovars, z. B. mittels einer Zystenpunktion als Prophylaxe einer erneuten Torsion. Mit einer zügigen Intervention lassen sich die meisten Torsionen problemlos behandeln. Eine verzögerte operative Intervention führt meistens zu einem Verlust des ganzen Ovars.

Sollte eine Adnexektomie nach einer In-vitro-Fertilisation erforderlich sein, so ist eine Gestagensupplementation, z. B. mit mikronisiertem Progesteron, vaginal bis zum Abschluss des 1. Trimenons zu empfehlen.

\section{Korrespondenzadresse}

Prof. Dr. M. von Wolff

Frauenklinik/Abteilung für Gynäkologische Endokrinologie und Reproduktionsmedizin, Universitätsfrauenklinik Bern

Effingerstr. 102, 3010 Bern

Schweiz

michael.vonwolff@insel.ch

Interessenkonflikt. Der korrespondierende Autor gibt an, dass kein Interessenkonflikt besteht.

\section{Literatur}

1. Rackow BW, Patrizio P (2007) Successful pregnancy complicated by early and late adnexal torsion after in vitro fertilization. Fertil Steril 87:697.e9-12 